\title{
A SEQUENTIAL F. AND M. RIESZ THEOREM
}

\section{ELIZABETH ANN HEARD}

Introduction. Let $C$ and $D$ be the closed unit circle and the closed unit disc in the complex plane. Let $A$ be the sup-norm Banach algebra of complex-valued functions continuous on $D$ which are analytic in the interior of $D$; $\lambda$ is Lebesgue measure on $C$. A function $f$ is in $H^{\infty}$ if it is bounded and analytic on the interior of $D$; such a function has boundary values almost everywhere on $C$ defined by $f\left(e^{i x}\right)$ $=\lim _{r \rightarrow 1} f\left(r e^{i x}\right)$. The following result has been announced by J.-P. Kahane:

THEOREM. If $\left\{g_{n}: n=1,2, \cdots\right\}$ is a sequence in $L^{1}(d \lambda)$ such that $L(f)=\lim \int f g_{n} d \lambda$ exists for all $f$ in $H^{\infty}$, then there is a $g$ in $L^{1}(d \lambda)$ such that $L(f)=\int f g d \lambda$ for all $f$ in $A$ and every weak $*$ cluster point $\mu$ of the measures $\mu_{n}$, where $d \mu_{n}=g_{n} d \lambda$, is absolutely continuous with respect to Lebesgue measure $(\mu \ll \lambda)$.

This theorem implies the classical theorem of F. and M. Riesz on analytic measures, for suppose $\mu$ is a measure on $C$ and $\int e^{\text {int }} d \mu(t)=0$ for $n=1,2, \cdots$. If $\left\{\sigma_{n}(t): n=1,2, \cdots\right\}$ is the sequence of Cesaro means of the Fourier series of $\mu$, then $\mu$ is the unique weak * cluster point of the set of measures $\left\{\mu_{n} ; d \mu_{n}=\sigma_{n} d \lambda, n=1,2, \cdots\right\}$ [3, p. 20], and $\lim \int f \sigma_{n} d \lambda=\int f d \lambda \int d \mu$ for all $f$ in $H^{\infty}$.

Kahane's argument is based on the fact that every closed subset $E$ of $C$ with zero Lebesgue measure is a peak set for the algebra $A$, that is, there is an element $f$ of $A$ such that $f(E)=1$ and $|f(x)|<1$ for all $x$ in $C \backslash E$. This fact follows from the F. and M. Riesz theorem on the circle. Since Kahane's theorem is equivalent to the F. and M. Riesz theorem on the circle, we call it a sequential F. and M. Riesz theorem. We intend to show that whenever there is a general F. and M. Riesz theorem there is also a sequential version.

Definitions. $X$ is a compact Hausdorff space; $C(X)$ the usual Banach space of all continuous, complex-valued functions on $X$ with the supremum norm; $M(X)$ is the class of all finite, complex, regular Borel measures on $X$, the dual of $C(X)$. For a subspace $S$ of $C(X), S^{-}$ is the set of all $\mu$ in $M(X)$ such that $\int f d \mu=0$ for all $f$ in $S$.

Let $m$ be a positive measure in $M(X)$ and $B$ a closed subspace of $C(X) . B$ and $m$ are said to satisfy the general F. and M. Riesz theorem if $\mu$ in $B^{\perp}$ implies $\mu \ll m$. Denote by $Q_{B}$ the weak $*$ closure of $B$ in $L^{\infty}(d m)$.

Received by the editors September 15, 1966. 
Theorem. If $B$ is a closed subspace of $C(X)$ and $m$ is positive in $M(X)$, then I implies II:

I. $\mu$ in $B^{\perp}$ implies $\mu \ll m$.

II. If $\left\{g_{n}: n=1,2, \cdots\right\}$ is a sequence in $L^{1}(d m)$ and $L(f)$ $=\lim \int f g_{n} d m$ exists for all $f$ in $Q_{B}$, then any representative $\mu$ of a coset $\mu+B^{\perp}$ which is a weak * cluster point of the set of cosets $\left\{\mu_{n}+B^{\perp}: d \mu_{n}\right.$ $\left.=g_{n} d m, n=1,2, \ldots\right\}$ in $M(X) / B^{\perp}$ is absolutely continuous with respect to $m(\mu \ll m)$ and there exists a function $g$ in $L^{1}(d m)$ such that $L(f)=\int f g d m$ for all $f$ in $B$.

Proof. Suppose that $\left\{g_{n}: n=1,2, \cdots\right\}$ is a sequence of functions in $L^{1}(d m)$ such that $\lim \int f g_{n} d m$ exists for all $f$ in $Q_{B}$. Let $\mu_{n}$ be the measure defined by $d \mu_{n}=g_{n} d m$ for $n=1,2, \cdots$. The principle of uniform boundedness shows the set $\left\{\mu_{n}+B^{\perp}: n=1,2, \cdots\right\}$ is uniformly bounded in $M(X) / B^{\perp}$. By weak * compactness of the unit ball of $M(X) / B^{\perp}$ there is a coset $\mu+B^{\perp}$ which is a cluster point of the set $\left\{\mu_{n}+B^{\perp}: n=1,2, \cdots\right\}$. Let $\mu$ be any representative of the coset $\mu+B^{\perp}$. Then $\lim \int f d \mu_{n}=\int f d \mu=L(f)$ for all $f$ in $B$. We claim $\mu$ is absolutely continuous with respect to $m$. Suppose this is not so. Since $\mu$ is a regular measure there exists a closed set $E$ such that $m(E)=0$ and $\mu(E) \neq 0$.

We require a sequence of functions $\left\{f_{n} \in B: n=1,2, \cdots\right\}$ having the following properties:

(i) $\left\|f_{n}\right\|<1+2^{-n}$,

(ii) $f_{n}(x)=1$ for all $x$ in $E$,

(iii) $\lim f_{n}=0$ a.e. $d m$,

(iv) $\lim f_{n}=C_{E}$ a.e. $d|\mu|$ where $C_{E}$ is the characteristic function of the set $E$,

(v) $f=\sum_{j=1}^{\infty}(-1)^{j} f_{n(j)}$ is in $Q_{B}$ for every strictly increasing sequence of positive integers $\{n(j) ; j=1,2, \cdots\}$.

The construction of this sequence $\left\{f_{n}\right\}$ is by induction. Let $\left\{U_{1}, U_{2}, \cdots\right\}$ be a sequence of open subsets of $X$ such that $E \subset U_{n+1}$ $\subset U_{n} ; m\left(U_{n}\right)<2^{-n}$; and $|\mu|\left(U_{n} \backslash E\right)<2^{-n}$ for all positive integers $n$. Let $E_{1}=U_{1}$. By Urysohn's lemma there is a real continuous function $d_{1}$ on $X$ such that $0 \leqq d_{1} \leqq 1, d_{1}(E)=1$ and $d_{1}\left(X \backslash E_{1}\right)=0$. The assumption that $I$ is true and the general Rudin-Carleson theorem [1] allow us to choose a function $f_{1}$ in $B$ such that $f_{1}(E)=1$ and $\left|f_{1}\right|<d_{1}+2^{-1}$.

Now suppose that $f_{1}, \cdots, f_{n}$ and the sets $E_{1}, \cdots, E_{n}$ which are open neighborhoods of $E$ are chosen. Let $V_{n+1}$ be the set of all $x$ in $X$ for which $\left|f_{n}-1\right|<2^{-n}$. Set $E_{n+1}=E_{n} \cap V_{n+1} \cap U_{n+1}$. There is a real function $d_{n+1}$ on $X$ such that $0 \leqq d_{n+1} \leqq 1, d_{n+1}(E)=1$, and $d_{n+1}\left(X \backslash E_{n+1}\right)=0$. Using Bishop's theorem, we choose a function $f_{n+1}$ in $B$ such that $f_{n+1}(E)=1$ and $\left|f_{n+1}\right|<d_{n+1}+2^{-(n+1)}$. 
Now we wish to show that the sequence $\left\{f_{n}\right\}$ satisfies (i)-(v). Properties (i) and (ii) are clear, $\lim f_{n}=0$ except on the set $F=\bigcap_{j=1}^{\infty} E_{j}$ and $m(F)=0$ since $m(F) \leqq m\left(E_{n}\right) \leqq m\left(U_{n}\right)<2^{-n}$ for all integers $n$, so (iii) is true. Now $\lim f_{n}=C_{E}$ except on the set $F \backslash E$ but $|\mu|(F \backslash E)$ $\leqq|\mu|\left(E_{n} \backslash E\right) \leqq|\mu|\left(U_{n} \backslash E\right)<2^{-n}$, so that $|\mu|(F \backslash E)=0$ and $\lim f_{n}$ $=C_{E}$ a.e. $d|\mu|$ (iv).

It remains to show that $(\mathrm{v})$ is true. Let $\{n(j): j=1,2, \cdots\}$ be any strictly increasing sequence of positive integers. Let $E_{n(0)}=X$. Notice that $F=\bigcap_{j=1} E_{j}=\bigcap_{j=1}^{\infty} E_{n(j)}, X \backslash F=\bigcup_{j=1}^{\infty}\left(E_{n(j-1)} \backslash E_{n(j)}\right)$ and that the sets in this union are pairwise disjoint. Suppose $q$ is in the set $E_{n(k)} \backslash E_{n(k+1)}$. Then $q$ is in $\bigcap_{j=1}^{\boldsymbol{k}-1} E_{n(j)+1}$, and in $\bigcap_{j=k+1}^{\infty} X \backslash E_{n(j)}$. Therefore:

$$
\left|\sum_{j=1}^{k-1}(-1)^{j} f_{n(j)}(q)\right| \leqq \sum_{j=1}^{k-1}\left|\left(f_{n(j)}(q)-1\right)(-1)^{j}\right|+\left|\sum_{j=1}^{k-1}(-1)^{j-1}\right|
$$

$$
\leqq \sum_{j=1}^{k-1} 2^{-n(j)}+1
$$

$$
\begin{aligned}
\left|\sum_{j=k+1}^{\infty}(-1)^{j} f_{n(j)}(q)\right| & \leqq \sum_{j=k+1}^{\infty}\left(d_{n(j)}(q)+2^{-n(j)}\right) \leqq 2^{-k}, \\
\left\|f_{n(k)}\right\| & \leqq 1+2^{-n(k)} .
\end{aligned}
$$

Hence the series $f(q)=\sum_{j=1}^{\infty}(-1)^{j} f_{n(j)}(q)$ converges and the absolute nature of the inequalities (A), (B) and (C) shows that the sequence $\left\{s_{n}(q): s_{n}(q)=\sum_{j=1}^{n}(-1)^{j} f_{n(j)}(q), n=1,2, \cdots\right\}$ converges to a value $f(q)$ and $\left|s_{n}(q)\right|<4$ for all positive integers $n$. Thus $f(q)$ is defined for all $q$ in the set $X \backslash F$. Let $z$ be in $F$. Then $z$ is in $E_{n(j)+1}$ for $j=1,2, \cdots$ and

$$
s_{n}(z)=\sum_{j=1}^{n}(-1)^{j} f_{n(j)}(z)=\sum_{j=1}^{n}(-1)^{j}\left(f_{n(j)}-1\right)+\sum_{j=1}^{n}(-1)^{j-1},
$$

so that

$$
\left|s_{n}(z)\right| \leqq \sum_{j=1}^{n}\left|f_{n}(j)(z)-1\right|+\left|\sum_{j=1}^{n}(-1)^{j-1}\right| \leqq \sum_{j=1}^{n} 2^{-n(j)}+1<4 .
$$

Therefore $f(x)=\lim s_{n}(x)$ is the pointwise limit almost everywhere $d m$ of a uniformly bounded sequence of functions contained in $B, f$ is in $Q_{B}$, and ( $\mathrm{v}$ ) is shown.

The remainder of the proof parallels Kahane's argument. Q.E.D.

It is not yet proved whether or not the limit relation of Kahane's theorem

$$
L(f)=\int f g d \lambda,
$$


holds for all $f$ in $H^{\infty}$. (An affirmative answer would show that $L^{1} / H_{0}^{1}$ is weakly sequentially complete.) Kahane showed that it holds for a good many functions in $H^{\infty}$; that is, in addition to functions in $A$ it holds for almost all translates of functions in $H^{\infty}$. The following argument suggested by James Wells shows that it holds for certain functions in $H^{\infty} \backslash A$.

THEOREM. Let $E$ be a closed subset of the unit circle with $\lambda(E)=0$. If the hypotheses of Kahane's theorem hold, then

$$
\lim \int f g_{n} d \lambda=\int f g d \lambda
$$

for every $f$ in $H^{\infty}$ which is continuous at all points of the closed unit disc, except possibly at the points of $E$.

Proof. Let $f$ be a function in $H^{\infty}$ which is continuous off $E$. We claim $L(f)=\int f g d \lambda$. If $G$ is any function in $A$ which vanishes on $E$

$$
\lim \int G f g_{n} d \lambda=\int G f g d \lambda .
$$

Also $\lim \int F f g_{n} d \lambda$ exists for all $F$ in $H^{\infty}$, so there exists $\phi$ in $L^{1}(d \lambda)$ such that

$$
\lim \int h\left(f g_{n}\right) d \lambda=\int h \phi d \lambda
$$

for all $h$ in $A$. Then $\int h(f g-\phi) d \lambda=0$ for all $h$ in $A$ which vanish on the set $E$.

Let $h$ be a fixed function in $A$ which is zero on $E$ and has no other zeros on the closed unit disc. For $p=1,2, \cdots, h^{1 / p}$ belongs to $A$ and $\int h^{1 / p}(f g-\phi) d \lambda=0$. The sequence $\left\{h^{1 / p}: p=1,2, \cdots\right\}$ is uniformly bounded on the closed unit disc and $\lim h^{1 / p}=1$ a.e. Therefore $\int(f g-\phi) d \lambda=0$. By letting $h=1$ in $(*)$ we see

$$
\int f g d \lambda=\int \phi d \lambda=\lim \int f g_{n} d \lambda=L(f) . \quad \text { Q.E.D. }
$$

\section{REFERENCES}

1. E. Bishop, A general Rudin-Carleson theorem, Proc. Amer. Math Soc. 13 (1962), 140-143.

2. I. Glicksberg, Measures orthogonal to algebras and sets of antisymmetry, Trans. Amer. Math. Soc. 105 (1962), 415-435.

3. K. Hoffman, Banach spaces of analytic functions, Prentice-Hall, Englewood Cliffs, N. J., 1962.

4. J.-P. Kahane, Another theorem on bounded analytic functions, Proc. Amer. Math. Soc. 18 (1967), 827-831. 